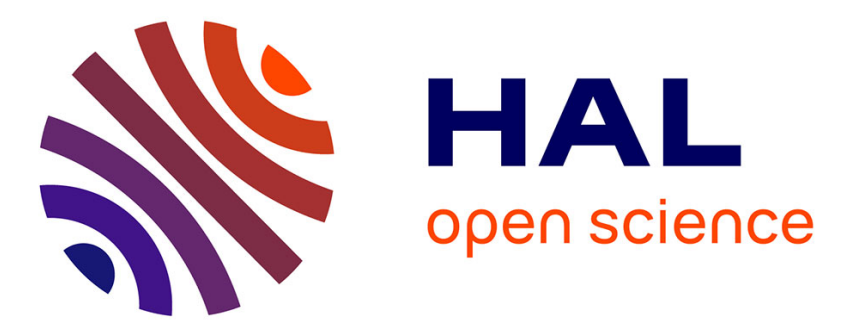

\title{
Mécanosynthèse de composés et de composites nanocristallins
}

Gérard Le Caër, R. de Araujo Pontès, D. Osso, S. Bégin-Colin, P. Matteazzi

\section{To cite this version:}

Gérard Le Caër, R. de Araujo Pontès, D. Osso, S. Bégin-Colin, P. Matteazzi. Mécanosynthèse de composés et de composites nanocristallins. Journal de Physique IV Proceedings, 1994, 04 (C3), pp.C3-233-C3-241. 10.1051/jp4:1994332 . jpa-00252529

\section{HAL Id: jpa-00252529 https://hal.science/jpa-00252529}

Submitted on 1 Jan 1994

HAL is a multi-disciplinary open access archive for the deposit and dissemination of scientific research documents, whether they are published or not. The documents may come from teaching and research institutions in France or abroad, or from public or private research centers.
L'archive ouverte pluridisciplinaire HAL, est destinée au dépôt et à la diffusion de documents scientifiques de niveau recherche, publiés ou non, émanant des établissements d'enseignement et de recherche français ou étrangers, des laboratoires publics ou privés. 


\title{
Mécanosynthèse de composés et de composites nanocristallins
}

\author{
G. LE CAËR, R. DE ARAUJO PONTĖS, D. OSSO, S. BÉGIN-COLIN et P. MATTEAZZI* \\ Laboratoire de Science et Génie des Matériaux Métalliques, URA 159 du CNRS, Ecole des Mines, \\ 54042 Nancy cedex, France \\ * Dipartimento di Scienze e Tecnologie Chimiche, Università di Udine, Via Cotonificio 108, 33100 Udine, \\ Italie
}

Résumé: La mécanosynthèse d'alliages $\mathrm{Fe}-\mathrm{Ni}$ et $\mathrm{Cr}-\mathrm{Sn}$, du carbure $\mathrm{Fe}_{7} \mathrm{C}_{3}$, de nanocomposites alumine-métal et d'oxydes est décrite. Les hétérogénéités des poudres en cours de broyage, les défauts des nanomatériaux ainsi élaborés, les propriétés magnétiques de solutions solides $\mathrm{Cr}-\mathrm{Sn}$, les mécanismes de synthèse des nanocomposites sont caractérisés par spectrométrie Mössbauer. L'influence de la nature des matériaux de broyage est mise en évidence dans le cas des oxydes.

Abstract : Mechanical alloying of alloys like $\mathrm{Fe}-\mathrm{Ni}, \mathrm{Cr}-\mathrm{Sn}$, of $\mathrm{Fe}_{7} \mathrm{C}_{3}$ carbide, synthesis of alumina-metal nanocomposites by reactive milling and grinding of oxydes is described. Heterogeneities of powders during grinding, defects of synthesized nanomaterials, magnetic properties of Cr-Sn solid solutions, mechanisms of synthesis of nanocomposites are characterized by Mössbauer spectroscopy. The influence of the materials constituting the grinding media is displayed in the case of oxides.

\section{1 - Introduction}

Le broyage est sans aucun doute l'une des activités les plus anciennes de l'homme. II constitue maintenant un nouveau moyen d'élaboration de matériaux. La mécanosynthèse ("mechanical alloying") /1/ se définit comme un procédé de synthèse par cobroyage, en général à sec, de mélanges de poudres d'éléments purs ou combinés dans un broyeur à haute énergie. L'énergie mécanique fournie à la charge (poudres + billes) est telle que les billes sont dans leur grande majorité et en permanence en mouvement relatif /1/. Limitée d'abord à l'élaboration de superalliages à dispersion d'oxydes, la mécanosynthèse connaît maintenant un développement considérable en raison de l'intérêt porté aux nanomatériaux et plus généralement aux matériaux métastables /2/. La mécanochimie /3/ étudie par ailleurs les modifications de réactivité chimique des solides après ou au cours d' un traitement mécanique. L'élaboration de matériaux par voie mécanique peut ainsi mettre en oeuvre une réaction à l'état solide menée jusqu'à son terme, une activation sans réaction, avec par exemple formation de structures lamellaires et introduction de défauts, suivie d'un traitement thermique $/ 3,4 /$, diverses réactions chimiques entre poudres cobroyées ou entre poudres et atmosphère de broyage /5-7/, diverses transformations structurales ou chimiques induites par le broyage. De nombreux travaux tentent en outre de modéliser la physique du procédé, de comprendre les mécanismes de synthèse, d'expliquer l'état final du mélange à partir de diagrammes d'équilibre dynamique ou métastable et de caractériser la structure et les propriétés des matériaux ainsi produits $/ 8-17 /$. Nous avons récemment étudié la synthèse de nanomatériaux divers : carbures et siliciures par broyage direct des éléments avec le carbone ou le silicium /6,18-19/, composites métal-oxyde /20/ ou métal-sulfure /21/. Le présent article décrit quelques contributions à l'étude des mécanismes de mécanosynthèse 
et à une meilleure connaissance des nanomatériaux grâce, en particulier, à l'utilisation de la spectrométrie Mössbauer.

\section{2 - Méthodes expérimentales}

Deux broyeurs ont été mis en oeuvre : un broyeur planétaire (Fritsch Pulvérisette, 7 billes ) et un broyeur vibrant (Spex 8000, 2 billes) avec des jarres étanches et des billes en acier ou en carbure de tungstène lorsqu'il est nécéssaire d'éviter une pollution réactive des matériaux élaborés. Le broyage de la zircone est effectué avec des matériaux de broyage en zircone 122/. La préparation de la jarre et de son contenu ( poudres + billes) prêts au broyage s'effectue dans une boîte à gants sous atmosphère d'argon. Le rapport $R$ de la masse de poudre à la masse de billes est de l'ordre de $1 / 40$ pour le broyeur planétaire et de $1 / 10$ pour le broyeur vibratoire. Le broyage est opéré de façon discontinue : chaque heure et demie de broyage est suivie d'une période d'arrêt de 30 minutes. Dans la suite, nous noterons $p-A_{x} B y$ le mélange initial des poudres de $A$ et des poudres de $B$ dans les proportions atomiques $x$ et y $(A, B=$ éléments ou composés). Les échantillons broyés sont caractérisés par diffraction des rayons $X\left(C_{0} K_{\alpha 1}, \lambda=0.17889 \mathrm{~nm}\right)$, microscopies électroniques à balayage et à transmission et, lorsque cela est possible, par spectrométrie Mössbauer ( $57 \mathrm{Fe}$ et $\left.{ }^{119} \mathrm{Sn}\right)$.

\section{3 - Alliages et Composés}

Des hétérogénéités de composition et donc le plus souvent structurales prennent à l'évidence place mais surtout persistent dans les poudres lors de la mécanosynthèse d'alliages ou de composés à partir des poudres élémentaires. Ces hétérogénéités sont particulièrement bien observées par spectrométrie Mössbauer au cours de l'élaboration d' alliages $\mathrm{Fe}_{1-x \mathrm{Ax}}$ dont les propriétés magnétiques varient sensiblement en fonction de la concentration, tels que Fe-V, Fe-Sn, Fe-Si , Fe-Ni /23-25/. Par exemple, du fer non allié est détecté pratiquement jusqu'à la formation complète de l'alliage Fe0.5 0.5 ou du composé intermétallique FeSi. Ces fluctuations ne dépendent pas de la taille des particules. Elles doivent être prises en compte dans l'interprétation des différentes mesures effectuées pour caractériser les poudres au cours du broyage car elles peuvent fausser les paramètres déduits de ces mesures (tailles de cristallites, paramètres de maille, etc..). Ces hétérogénéités peuvent amener à préférer dans certains cas une synthèse par broyage de poudres d'une phase ou d'un mélange de phases qui a la composition moyenne du matériau visé plutôt que de broyer un mélange des poudres des éléments.

\section{1 - Mécanosynthèse d'alliages FeNi}

Les alliages cubiques à faces centrées $\mathrm{Fe}_{1-\mathrm{x}} \mathrm{Ni} \times(0.25 \leq \mathrm{x} \leq 0.50)$ jouent un grand rôle en astrophysique car ils sont présents dans la plupart des météorites. Une lacune de solubilité d'origine magnétique explique la séparation de phase observée dans des météorites refroidis pendant des millions d'années. Reuter et al. /26/ ont cependant montré qu'un équilibre thermodynamique n'est jamais atteint au-dessous de $-673 \mathrm{~K}$. La décomposition d'alliages C.F.C. est impossible dans les conditions usuelles de synthèse à cause des très faibles valeurs des coefficients de diffusion. Ainsi, le coefficient d'interdiffusion est de l'ordre de $\quad 10^{-31} \mathrm{~m}^{2} / \mathrm{s}$ à $600 \mathrm{~K}$. A la température ambiante, le champ hypertin moyen est supérieur à celui du fer $\alpha(331 \mathrm{kG})$ dans le domaine $\alpha$ alors qu'il est nul ou faible pour les alliages C.F.C. qui ont une teneur en $\mathrm{Ni}$ de l'ordre de 0.30 et qu'il reste inférieur à environ $310 \mathrm{kG}$ dans tout le domaine $\gamma$ jusqu'à $x=1$. Deux alliages ont été élaborés pour des concentrations $x=0.36$ et $x=0.50 / 25 /$. Pour $x=0.36$, les tailles moyennes des cristallites $<d>$ sont de $14 \mathrm{~nm}$ avec le broyeur vibrant pour un temps de broyage $t b \geq 24 \mathrm{~h}$ et de $15 \mathrm{~nm}$ pour $\mathrm{tb}=3 \mathrm{~h}$ avec le broyeur planétaire. Dans le dernier cas, $<d>$ diminue au cours d'un broyage profongé et vaut $9 \mathrm{~nm}$ pour $\mathrm{tb}=9 \mathrm{~h}$. Les larges fluctuations de concentration sont bien visibles sur les spectres Mössbauer (figure 1). Trois composantes principales coexistent lors de la synthèse (figure 1, 
$t_{b}=12 h$ ) : la première avec un champ hyperfin voisin de $330 \mathrm{kG}$ est caractéristique du fer $\alpha$ (figure $3 a$ ) et des alliages C.C., la seconde (flèches extérieures sur la figure 1) est associée aux alliages C.F.C. magnétiques avec des champs inférieurs à $310 \mathrm{kG}$, la troisième (flèche centrale), paramagnétique avec un déplacement isomérique de $0.1 \mathrm{~mm} / \mathrm{s}$, est associée aux alliages C.F.C. avec $x \sim 0.30$. La synthèse est terminée après $24 \mathrm{~h}$ de broyage dans le broyeur vibrant. La composante centrale a complètement disparu (figure 1). Les distributions de champ hyperfin /25/ montrent un pic principal pour $\mathrm{H}=306 \mathrm{kG}$ ainsi qu'un épaulement situé autour de $258 \mathrm{kG}$ pour $\mathrm{x}=0.36$. Le premier champ correspond bien au champ attendu pour l'alliage Fe0.64Nio.36. La distribution s'élargit et l'intensité de la seconde composante augmente au cours d'un broyage prolongé dans le broyeur planétaire en même temps que diminue $<d>$. Il paraît donc raisonnable d'attribuer cette seconde composante à des atomes de fer situés dans les zones interfaciales de ces matériaux nanocristallins. II est intèressant de souligner qu'une telle synthèse ne pourrait pas avoir lieu si l'on plaçait le mélange de poudres dans un four à une température égale à la température maximum $T_{M}$ atteinte lors du broyage. Une estimation raisonnable est $T_{M}<700 \mathrm{~K} / 10 /$. Une limite inférieure du coefficient de diffusion effectif peut être obtenue en supposant que la diffusion se produit pendant le temps tb et que la profondeur de pénétration est de $10 \mathrm{~nm}$. Pour tb=3 et $24 \mathrm{~h}$, nous calculons $D_{\text {eff }} \sim 10^{-22}-10^{-23} \mathrm{~m}^{2} / \mathrm{s}$. Le coefficient de diffusion précédent est alors supérieur d'au moins 5-6 ordres de grandeur au coefficient de diffusion thermique à $673 \mathrm{~K}$. L'accélération considérable de la diffusion peut être attribuée à la forte densité de joints de grains et de défauts créés par le broyage. II pourrait être intèressant d'étudier l'influence de l'activation par broyage de mélanges de poudres de $\mathrm{Fe}$ et de $\mathrm{Ni}$ sur l'état structural final après recuit à température modérée.
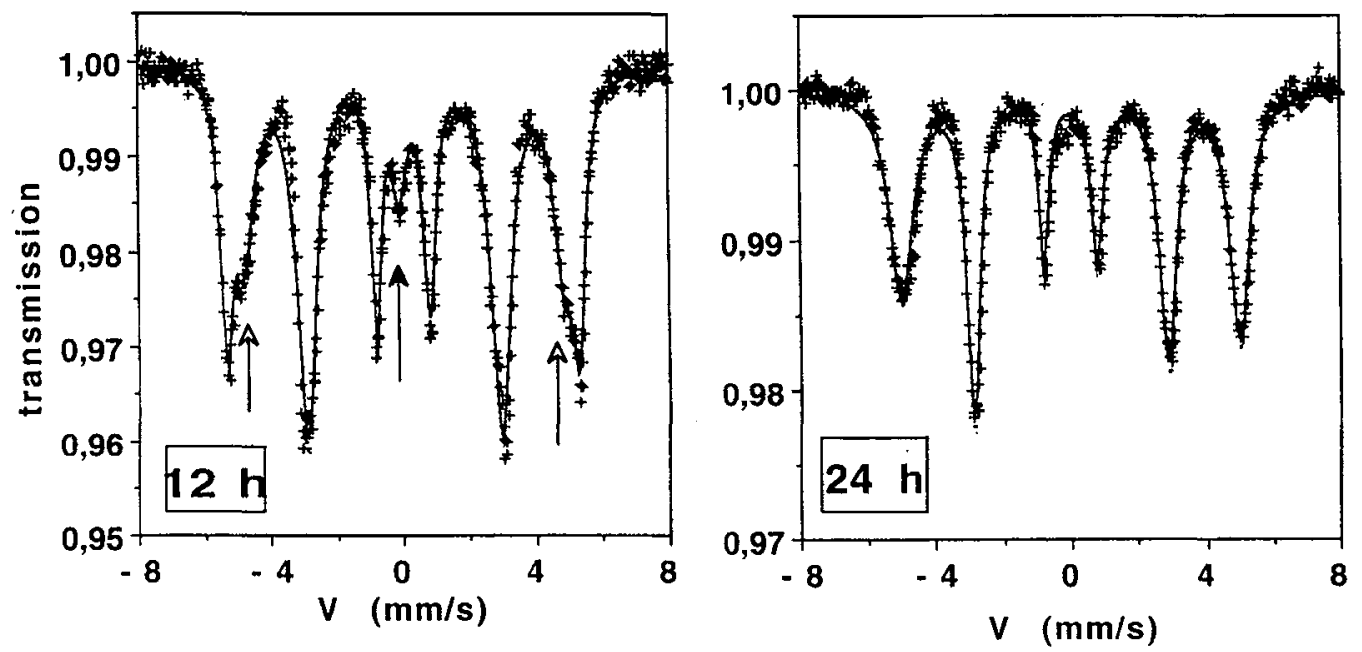

Figure1: Spectres Mössbauer à la température ambiante de poudres p-Fe0.5Ni0.5 broyées pendant 12 et $24 \mathrm{~h}$ dans un broyeur vibrant.

\section{2 - Mécanosynthèse de solutions solides étendues Cr-Sn}

Le chrome est l'archétype des solides antiferromagnétiques itinérants avec une température de Néel $T_{N}=311 \mathrm{~K}$. Au-dessous de $T_{N}$, lordre antiferromagnétique est dû à une onde de densité de spin (O.D.S.) statique, de vecteur d'onde dirigé selon [100], avec une amplitude incommensurable avec le paramètre du réseau cubique. Dans les alliages de chrome ou sous l'effet de contraintes, l'onde peut devenir commensurable. La diffraction neutronique, qui est la technique principale d'étude, est utilement complètée par diverses techniques hyperfines. Pour la spectrométrie Mössbauer, c'est l'isotope ${ }^{119} \mathrm{Sn}$ qui conduit aux résultats 
les plus intèressants mais il faut de l'ordre de 0.2 at\% pour avoir des spectres bien résolus $127 /$. Pour mieux connaître la perturbation apportée par l'étain, il peut être utile d'étudier des solutions solides plus concentrées $\mathrm{Cr}$-Sn. La solubilité de l'étain est mal connue, mais elle est inférieure à 1 at.\% /28/. Le diagramme d'équilibre ne comporte aucun composé intermétallique. Un composé métastable $\mathrm{Cr}_{2} \mathrm{Sn}_{3}$ a cependant été préparé par un processus d'amalgamation par Hollan et al. /29/. Le systéme Cr-Sn présente un intérêt supplémentaire pour la caractérisation de l'état nanocristallin : l'étain ségrégé aux joints de grains du chrome (et aussi du fer) ou même aux sous-joints (Dubiel,1987, résultats non publiés) a un champ hyperfin nul alors que les atomes d'étain des grains voient un champ non nul ou une distribution de champ. Notons tout d'abord que le composé intermétallique $\mathrm{Cr}_{2} \mathrm{Sn}_{3}$ est obtenu après $24 \mathrm{~h}$ de broyage de $\mathrm{p}-\mathrm{Cr}_{2} \mathrm{Sn}_{3}$ dans le broyeur vibrant avec des matériaux en WC. Après $t b=24 h$, des solutions solides C.C. étendues sont formées par broyage de $\mathrm{p}-\mathrm{Cr}_{1-\mathrm{x}} \mathrm{Sn}_{\mathrm{x}}$ (broyeur vibrant , WC, $\mathrm{x} \leq 0.10$ ). Le paramètre cristallin augmente pratiquement linéairement avec $x$ pour $x \leq 0.10$ (de 0.2897 pour $x=0.005$ à $0.2931 \mathrm{~nm}$ pour $x=0.10$ ), prouvant ainsi l'extension effective de la solution solide. La taille de grain finale est indépendante de $x$ et vaut $\sim 10 \mathrm{~nm}$. La solution solide Cro.90Sno.10, chauffée à une vitesse de $10 \mathrm{~K} / \mathrm{min}$ est stable jusqu'à $933 \mathrm{~K}$. Au-delà de $x=0.10$, se forme le composé intermétallique et une solution solide en proportions variables seion $x$. Des micrographies montrent en outre que la structure lamellaire initiale classiquement observée en mécanosynthèse de matériaux ductiles s'est formée à partir de chrome et d'étain fondu $/ 25 /$. La température locale atteint ainsi au moins la valeur de $505 \mathrm{~K}$. Les spectres Mössbauer (de Araujo Pontès, Le Caër et Calage, à paraître) sont ceux de composés non-magnétiques à la température ambiante au contraire de spectres d'alliages Cro.998Sno.002/27/. A $77 \mathrm{~K}$ les spectres ( $x$ de 0.03 à 0.10 ) sont constitués d'une raie centrale intense $(H=0)$ et d'une partie large (figure 2 ) qui montre qu'une fraction de l'échantillon est magnétique. La comparaison des spectres à 77 et $4.2 \mathrm{~K}$ montre de plus qu'une fraction des atomes d'étain à $\mathrm{H}=0$ voit un champ non-nul par abaissement de la température. Les échantillons sont magnétiquement hétérogènes. A $4.2 \mathrm{~K}$, le pic central représente $\sim 30 \%$ des atomes d'étain alors que le champ hyperfin moyen vaut $\langle H>=68 \mathrm{kG}$ pour $\mathrm{x}=0.05$ et $\mathrm{x}=0.10$. Les distributions de champ hyperfin montrent que le champ de $\sim 30 \%$ des atomes d'étain dépasse $100 \mathrm{kG}$ à $4.2 \mathrm{~K}$ pour $\mathrm{x} \geq 0.05$ et que le champ maximum est de l'ordre de $150 \mathrm{kG}$ au lieu de $100 \mathrm{kG}$ dans les solutions solides très diluées. Ces résultats sont en accord avec l'augmentation du moment magnétique du chrome avec le volume atomique prédite par des calculs théoriques $/ 30 /$. L'étain semble se comporter comme un trou magnétique. Le pic central s'explique pour partie par des phénomènes de relaxation liés aux tailles des grains de la solution solide et pour partie par les atomes d'étain situés aux joints de grains. Une étude des solutions solides à nanograins Fe-Sn est en cours.
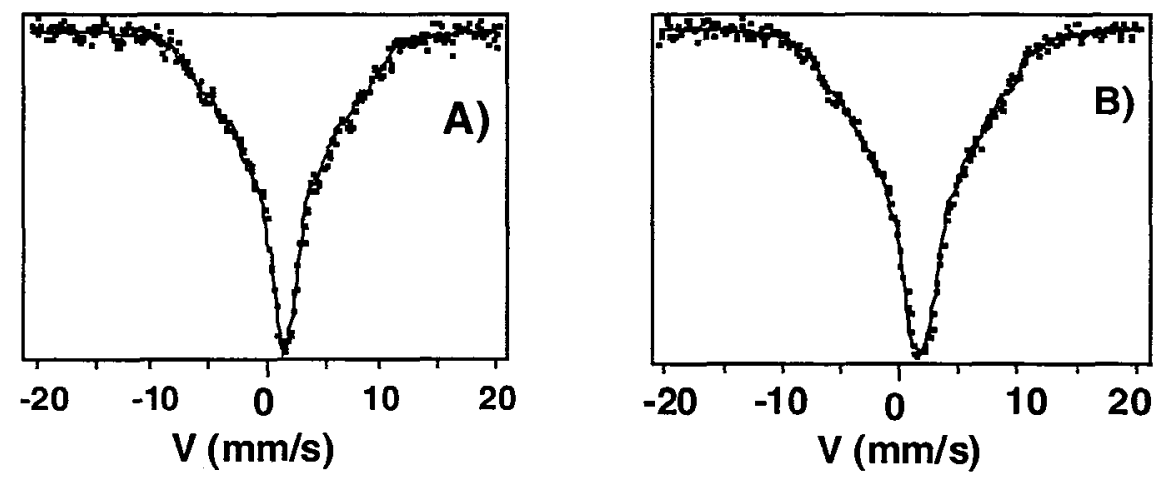

Figure 2 : Spectres Mössbauer à $77 \mathrm{~K}$ de la solution solide $\mathrm{Cr}_{1-\mathrm{x}} \mathrm{S} n_{\mathrm{x}}$ pour a) $\mathrm{x}=0.05 \mathrm{~b}$ ) $\mathrm{x}=0.10$ 


\section{3 - Mécanosynthèse du carbure $\mathrm{Fe}_{7} \mathrm{C}_{3}$}

Nous avons montré dans des articles précédents $/ 18,19$ / qu'il est possible de synthétiser presque tous les carbures par simple broyage des éléments à carburer et du carbone. Outre son intérêt historique et technologique, le système Fe-C fait toujours l'objet de nombreux travaux scientifiques. La mécanosynthèse de carbures de fer nanocristallins est attractive car les poudres permettent de préparer des composites fer-cémentite $\mathrm{Fe}_{3} \mathrm{C}$ consolidés et encore nanocristallins avec des duretés particulièrement élevées (> $1000 \mathrm{HV}$, Matteazzi et al. , à paraître). Le carbure orthorhombique, à chaînes de prismes de fer centrés par des atomes de carbone, $\mathrm{Fe}_{7} \mathrm{C}_{3}$, a un spectre Mössbauer à raies très larges qui est très semblable à celui du même carbure obtenu par cristallisation d'alliages amorphes $\mathrm{Fe}-\mathrm{C}$ (figure 4 de la référence $/ 31 /$ ). Ce dernier carbure est constitué de microdomaines ordonnés de dimension -5-10 nm (/31/ et références). Un spectre Mössbauer à raies fines est au contraire obtenu dans les conditions d'équilibre à haute température et à haute pression. L'influence des défauts et la dimension nanométrique des grains sont ainsi confirmées dans ce type de composés comme ils le sont dans la cémentite, à nappes de prismes, préparée par mécanosynthèse $118,19 /$. La mécanosynthèse de la céméntite comporte une première étape d'incubation ( $\sim 10$ min pour $R=1 / 40$ dans le broyeur planétaire) pendant laquelle se forme une structure lamellaire $\mathrm{Fe}-\mathrm{C} / 25 /$, suivie de la synthèse progressive de la cémentite qui coexiste avec un carbure hexagonal. La synthèse est terminée, dans les conditions expérimentales précédentes, en moins de $90 \mathrm{~min}$. Les raies élargies des diffractogrammes $X$ et des spectres Mössbauer confirment le caractére nanocristallin et la présence de perturbations des champs hyperfins à une échelle nanométrique. Nous avons de même cherché à suivre la synthèse du carbure $\mathrm{Fe}_{7} \mathrm{C}_{3}$ à partir de $\mathrm{p}-\mathrm{Fe}_{7} \mathrm{C}_{3}$ dans un broyeur planétaire avec $R=1 / 40$ et non à partir de $p-F e C$ comme dans /31/ (de Araujo Pontès et $G$. Le Caër, à paraître). Un temps d'incubation de $\sim 10-15$ minutes est à nouveau observé pendant lequel les raies de diffraction du fer s'élargissent et celles du graphite ne sont plus clairement visibles. Les spectres Mössbauer restent ceux du fer pur sans élargissement notable (figure $3 a)$. La deuxiéme étape $(30 \leq \mathrm{tb} \leq 90 \mathrm{~min})$ voit se former un carbure nanocristallin , cémentite ou structure voisine à nappes de prismes un peu plus riche en carbone $/ 32 /$, jusqu'à disparition totale du fer (figure $3 b$ ). Le carbure à nappes de prismes se transforme progressivement en $\mathrm{Fe}_{7} \mathrm{C}_{3}$ au cours de la dernière étape $\left(90 \leq t_{\mathrm{b}} \leq 180 \mathrm{~min}\right.$, figure $3 c$ ). Un modèle possible d'une telle transformation, qui n'avait pas été observée à notre connaissance, a été proposé en 1971 par Schmitt /33/. Le modèle consiste en la répétition périodique d'un glissement d'une partie du réseau de la cémentite, qui conduit à une structure lacunaire du carbure $\mathrm{Fe}_{7} \mathrm{C}_{3}$, suivie d'une diffusion de carbone . La forte densité des défauts, présents dans le carbure à nanograins, doit jouer un rôle important dans cette transformation.
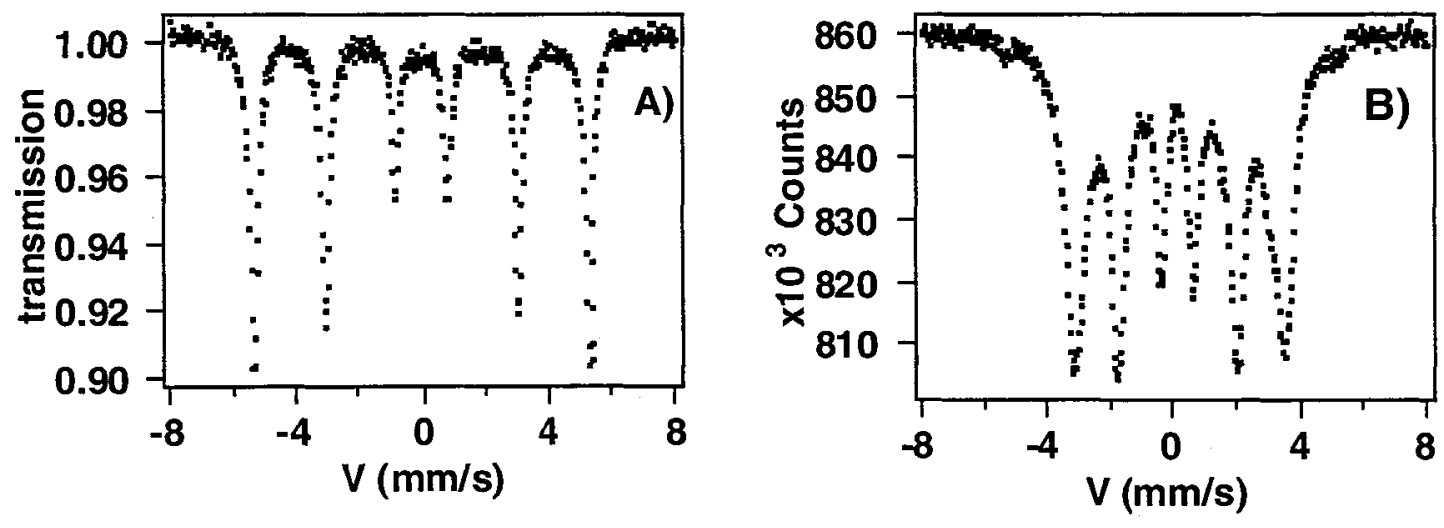


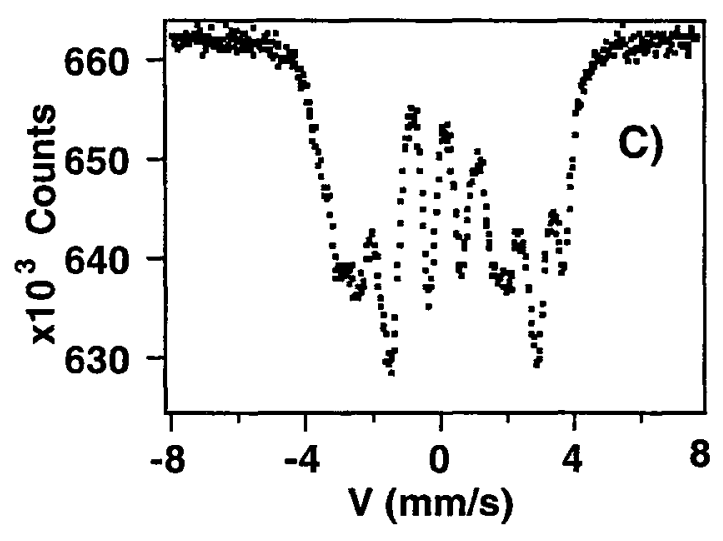

Figure 3 : Spectres Mössbauer à la température ambiante de poudres $\mathrm{p}-\mathrm{Fe}_{7} \mathrm{C}_{3}$ broyées pendant :
A) 15 minutes
B) 1 h30
C) $3 \mathrm{~h}$

\section{4 - Composites oxyde-métal et Oxydes}

Le broyage constitue un moyen de synthèse de matériaux divers par la mise en oeuvre de réactions d'échange d'éléments entre réactifs $120,34 /$. Les modifications structurales et chimiques induites par le broyage permettent de mettre en évidence l'importance de la nature des matériaux constitutifs des jarres et des billes dans le cas des oxydes.

\section{1 - Composites $\mathrm{Al}_{2} \mathrm{O}_{3}-(\mathrm{Fe}, \mathrm{Cr})$}

Le broyage réactif d'aluminium et d'oxydes de divers métaux permet de synthétiser des nanocomposites $\alpha-\mathrm{Al}_{2} \mathrm{O}_{3}$ - métal /20/. De tels composites peuvent aussi être obtenus par broyage direct de mélanges de poudres de $\alpha-\mathrm{Al}_{2} \mathrm{O}_{3}$ et des métaux et comparés aux précédents. Une réaction progressive ou une réaction brutale après un temps d'activation prennent place selon le système considéré et les conditions du broyage. Nous présenterons quelques résultats relatifs au système $\mathrm{Fe}-\mathrm{Cr} / 35 /$. Une teneur en chrome de $13 \%$ en poids, qui est celle de la jarre et des billes, a été choisie de façon à réduire l'effet de la pollution par les matériaux de broyage. Avec $\mathrm{R}=1 / 20$, l'oxyde de chrome $\mathrm{Cr}_{2} \mathrm{O}_{3}$ agit comme modérateur de la réduction de l'hématite $\mathrm{Fe}_{2} \mathrm{O}_{3}$. La diffraction des rayons $\mathrm{X}$ et la spectrométrie Mössbauer montrent que la réaction est totale au bout de 27 min (figure 4). Après $4 \mathrm{~h}$ de broyage, une analyse à la microsonde et les spectres Mössbauer montrent que le fer contient environ $10 \%$ at de $\mathrm{Cr}$ en solution. Le broyage direct de $\alpha-\mathrm{Al}_{2} \mathrm{O}_{3}, \mathrm{Fe}, \mathrm{Cr}$ (figure 5) pendant $4 \mathrm{~h}$ donne du fer avec moins de $3 \%$ at. de Cr. Une partie du chrome est donc restée sous forme métallique ou est passée dans l'alumine. La faible quantité de chrome et la largeur des raies de diffraction ne permettent pas de choisir. Comme dans le cas des composites $\alpha-\mathrm{Al}_{2} \mathrm{O}_{3}$ - $\mathrm{Fe}$, les spectres Mössbauer montrent l'existence d'une composante centrale non-magnétique et dissymétrique avec une enveloppe plus intense vers $\sim 0 \mathrm{~mm} / \mathrm{s}$ et une enveloppe moins intense, caractéristique de $\mathrm{Fe}^{2+}$ dans un environnement analogue à ceux de l'aluminium dans I' hercynite $\mathrm{FeAl}_{2} \mathrm{O}_{4}$, vers $\sim 2 \mathrm{~mm} / \mathrm{s}$ (figures 4 et 5). Le pic central peut être interprété comme la somme de diverses contributions $\mathrm{Fe}^{3+}$, $\mathrm{Fe}^{4+}$ et même amas de fer dans l'alumine $/ 36,37 /$. Le doublet asymétrique précédent est très semblable aux composantes centrales 

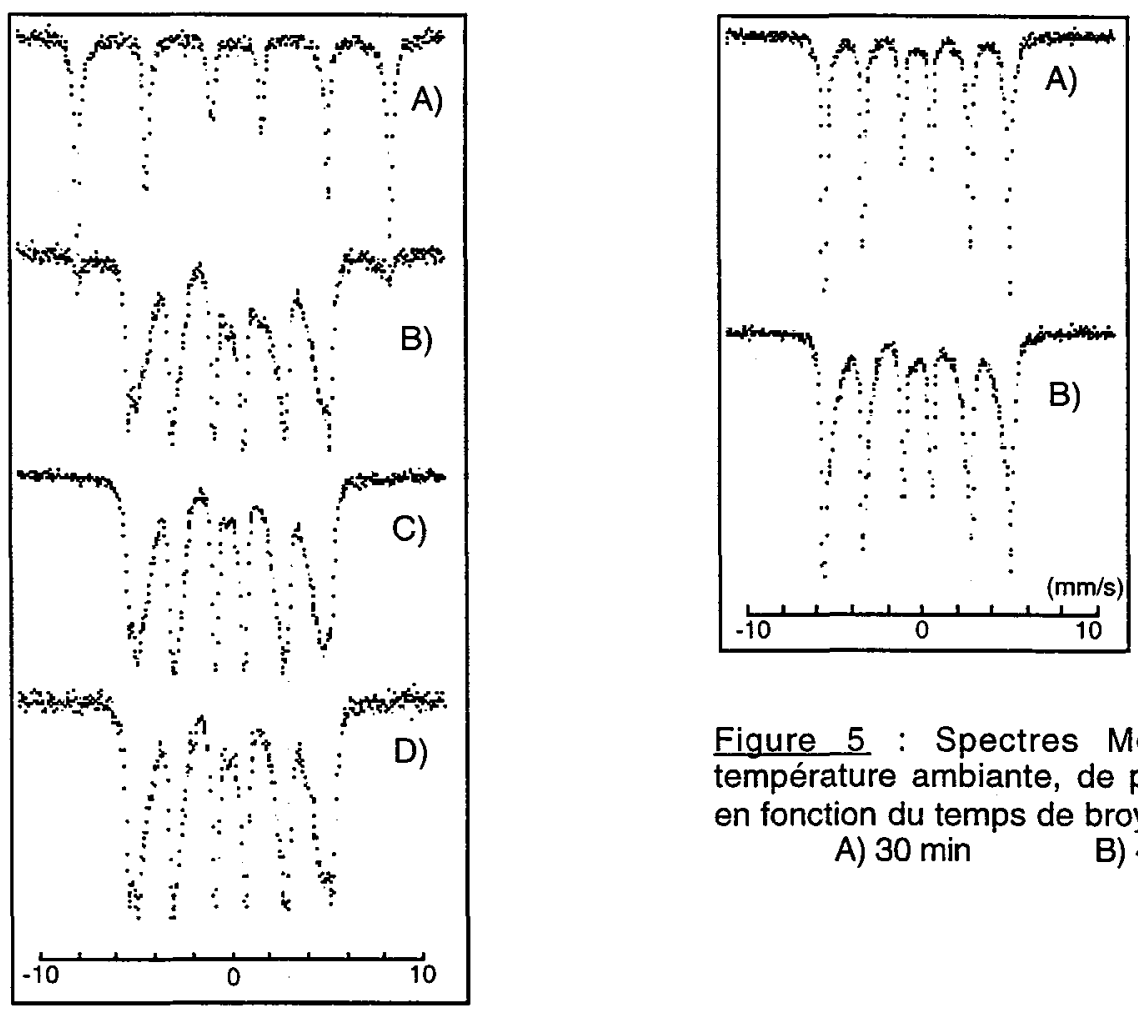

Figure 5 : Spectres Mössbauer, à la température ambiante, de $\mathrm{p}-\left(\mathrm{Al}_{2} \mathrm{O}_{3}, \mathrm{Fe}, \mathrm{Cr}\right)$ en fonction du temps de broyage :
A) $30 \mathrm{~min}$
B) $4 \mathrm{~h}$

Figure 4 : Spectres Mössbauer, à la température ambiante, de p-(Al, $\left.\mathrm{Fe}_{2} \mathrm{O}_{3}, \mathrm{Cr}_{2} \mathrm{O}_{3}\right)$ en fonction du temps de broyage : A) $8 \mathrm{~min}$

B) $15 \mathrm{~min}$

C) $27 \mathrm{~min}$

D) $4 \mathrm{~h}$

des spectres observées, dans certaines conditions, après implantation de fer dans $\alpha-\mathrm{Al}_{2} \mathrm{O}_{3}$ ou après mélange par faisceau d'ions de l'interface $\alpha-\mathrm{Al}_{2} \mathrm{O}_{3}-\mathrm{Fe} / 36,37 /$. Dans des composites $\alpha-\mathrm{Al}_{2} \mathrm{O}_{3}-\mathrm{Cr}$, la R.M.N. haute précision de ${ }^{27} \mathrm{Al}$ montre un pic principal à $10 \mathrm{ppm}$ qui correspond à un environnement octaédrique d'atomes d'oxygène pour les atomes d'aluminium. Un épaulement vers $30-40 \mathrm{ppm}$ est associé à des environnements à cinq atomes d'oxygène qui peuvent être attribués aux atomes d'aluminium des joints de grains 135/. La synthèse de composites $\alpha-\mathrm{Al}_{2} \mathrm{O}_{3}$ - Ti par broyage réactif de l'anatase $\mathrm{TiO}_{2}$ montre la formation transitoire de la forme orthorhombique haute pression de type $\alpha-\mathrm{PbO}_{2}$. Cette structure est également observée par broyage direct de l'anatase /38/ (paragraphe 4.2).

\section{2 - Oxydes}

La réduction de l'hématite en magnétite nanocristalline peut être induite par broyage avec une jarre et des billes en carbure de tungstène. Cette réduction est encore plus poussée si l'on ajoute du carbone à l'hématite de départ /39/. Après broyage de $\mathrm{SnO}_{2}$, pendant $1 \mathrm{~h}$ et $4 \mathrm{~h}$, dans un broyeur vibratoire avec des matériaux de broyage en acier, les billes sont recouvertes d'une couche uniforme d'étain métallique alors que la diffraction des rayons $X$ et la spectrométrie Mössbauer à $77 \mathrm{~K}$ ne permettent pas de détecter la présence de métal dans la poudre broyée. La mécanosynthèse de zircone dopée par du fer, à partir de mélanges p$\left(\mathrm{ZrO}_{2}, \mathrm{Fe}_{2} \mathrm{O}_{3}\right)$, conduit à du $\mathrm{Fe}^{3+}$ lorsque le broyage est effectué avec des matériaux en zircone et en grande majorité à du $\mathrm{Fe}^{2+}$ dans le cas de matériaux en carbure de tungstène 122/. Le broyage d'anatase $\mathrm{TiO}_{2}$ avec des matériaux en acier montre la formation immédiate 
et rapide de divers polymorphes, dont la forme haute pression de type $\alpha-\mathrm{PbO}_{2}$, suivie d'une transformation de cette dernière en rutile. Après $1 \mathrm{~h}$ de broyage, du rutile faiblement dopé par du $\mathrm{Fe}^{2+}$ est observé. Ce dernier état de valence est aussi obtenu par recuit de rutile en atmosphère réductrice (/38/ et références). Avec des rapports $R$ voisins mais des matériaux de broyage en alumine, Criado et al. /40/ observent au contraire la transformation directe de l'anatase en rutile. Les résultats précédents suggérent que la nature des matériaux de broyage a une grande importance en imposant des conditions réductrices ou non à l'interface jarre-oxyde et billes-oxyde. La réaction au contact poudre-matériau de broyage semble dans ce cas plus déterminante que la nature de l'atmosphère car les mêmes transformations structurales de l'anatase, avec pratiquement la même cinétique, sont observées lorsque le broyage est effectué dans l'air plutôt que dans l'argon. Une étude du broyage de l'anatase avec des matériaux en zircone est en cours. Les conditions physiques et chimiques devront donc être prises en compte pour prédire l'évolution de certains matériaux au cours du broyage.

\section{5 - Conclusion}

La caractérisation et la compréhension complète des nanomatériaux passe par la modélisation structurale des zones désordonnées que constituent les joints de grains. Cette modélisation sera aussi difficile et incertaine que celle des matériaux amorphes. Les techniques hyperfines, telles la spectrométrie Mössbauer ou la R.M.N., peuvent apporter des renseignements précieux sur la composition chimique et les propriétés magnétiques de ces zones interfaciales. Ces techniques sont des compléments utiles des méthodes de diffraction qui donnent des diffractogrammes à raies larges parfois difficiles à exploiter en raison de la taille des cristallites. La spectrométrie Mössbauer permet en outre d'étudier les mécanismes de synthèse, de caractériser le matériau élaboré avec ses défauts, de mettre en évidence la pollution par la jarre et les billes lorsqu'elles contiennent du fer. Elle pourra enfin contribuer à l'étude des propriétés des matériaux consolidés à nanograins que nous commençons à élaborer.

Remerciements: Nous remercions E. Bauer-Grosse, Y. Calage, D. Canet, P. Delcroix, A. Mocellin, A. Pianelli, P. Tekely, B. Vigneron, F. Wolf pour leur aide ainsi que pour d'utiles documents et discussions. Ce travail a bénéficié du soutien financier de la C.E.E. (Programme Science, contrat SC1-CT91-0668).

\section{REFERENCES}

/1/ BENJAMIN, J.S., Metall. Trans. 1 (1970) 2943

/2/ de BARBADILLO, J.J., Key Engineering Materials $77-78$ (1993) 187

/3/ BOLDYREV, V.V., J. de Chimie Physique 83 (1986) 821

14/ MALHOUROUX-GAFFET, N.et GAFFET, E., J. of Alloys and Compounds (1993) sous presse

15/ SCHAFFER, G.B. et McCORMICK, P.G., Metall. Trans. 21A (1990) 2789

/6/ MATTEAZZI, P., BASSET, D., MIANI, F. et LE CAER, G., Nanostructured Materials (1993) sous presse

/7/ CALKA, A., WILLIAMS, J.S. et MILLET, P., Scripta Metall. Mater. 27 (1992) 1853

/8/ CHEN, Y., Thèse, Université Paris-Sud, et Rapport CEA-R-5620 (1992)

19/ GAFFET, E., Mater. Sci. Eng. A 119 (1989) 185

110/ CHEN, Y., LE HAZIF, R. et MARTIN, G., Solid State Phenomena 23 \& 24 (1992) 271

/11/ LE BRUN, P., FROYEN, L. et DELAEY, L. , Mater. Sci. Eng. A161 (1993) 75

/12/ RYDIN, R.W., MAURICE, D. et COURTNEY, T.H., Metall. Trans. 24A (1993) 175

113/MARTIN, G. et BELLON, P., Metall. Sci. Technol. $\underline{9}$ (1991) 61

/14/ GERASIMOV, K.B., GUSEV, A.A., IVANOV, E.Y. et BOLDYREV, V.V., J. Mater. Sci. 26 (1991) 2495 
/15/ JOHNSON, W.L., Prog. Mater. Sci. 30 (1986) 81

/16/ YAVARI, A.R. et DESRE, P.J., Mat. Sci. Eng. A134 (1991)1315

/17/ GLEITER, H., Prog. Mater. Sci. 33 (1989) 223

/18/ LE CAER, G., MATTEAZZI, P., BAUER-GROSSE, E., PIANELLI, A. et BOUZY, E., J. Mater. Sci. 25 (1990) 4726

/19/ MATTEAZZI, P. et LE CAER, G. , J. Amer. Ceram. Soc. 74 (1991) 1382

/20/ MATTEAZZI, P. et LE CAER, G. , J. Amer. Ceram. Soc. 75 (1992) 2749

121/MATTEAZZI, P. et LE CAER, G. , Mat. Sci. Eng. A156 (1992) 229

122/ WOLF, F., Thèse d'I.N.P.L., Nancy , en cours de préparation

123/ FULTZ, B., LE CAER, G. et MATTEAZZI, P., J. Mater. Res. 4 (1989) 1450

124/ LE CAER, G. , MATTEAZZI, P. et FULTZ, B., J. Mater. Res. $\mathbf{Z}$ (1992) 1387

125/ de ARAUJO PONTES, R., Thèse d'I.N.P.L., Nancy (1992)

/26/ REUTER, K.B., WILLIAMS, D.B. et GOLDSTEIN, J.I., Metall. Trans. $20 A$ (1989) 719

/27/ DUBIEL, S.M. et LE CAER, G., Europhys. Lett. 4 (1987) 487

128/ DUBIEL, S.M., Phil. Mag. B 66 (1992) 551

129/ HOLLAN, L., LECOCQ, P. et MICHEL, A. C.R. Acad. Sc. Paris 258 (1964) 3309

/30/MORUZZI, V.L. et MARCUS, P.M., Phys. Rev. B 42 (1990) 8361

131/ LE CAER, G., MATTEAZZI, P., BAUER-GROSSE, E., FULTZ, B. et PIANELLI, A., J. de Physique (Paris) Colloque C4, 51 (1990) C4-151

132/ BAUER-GROSSE, E. et LE CAER, G. , Mater. Sci. Eng. 97 (1988) 273

133/ SCHMITT, B., Thèse d'Etat, Nancy (1971)

/34/ LIU, Y., DALLIMORE, M.P. et MCCORMICK, P.G., J. Magn. Magn. Mater. 116 (1992) L320 135/ OSSO, D., LE CAER, G., BEGIN-COLIN, S., MOCELLIN, A. et MATTEAZZI, P., EUROMAT93, Paris, 8-10 Juin 1993, sous presse

136/ McHARGUE, C.J., SKALD, P.S., FARLOW, G.C., PEREZ, A. et MAREST, G., J. Mater. Res. $\underline{6}$ (1991) 2145

137/ PEREZ, A., ABONNEAU, E., FUCHS, G., TREILLEUX, M., McHARGUE, C.J. et JOSLIN, D.L., Nucl. Instrum. Meth. B65 (1992) 129

138/ BEGIN-COLIN, S., LE CAER, G., MOCELLIN, A. et ZANDONNA, A, Phil. Mag. Lett. (1993) soumis

139/ MATTEAZZI, P. et LE CAER, G., Mater. Sci. Eng. A 149 (1991) 135

/40/ CRIADO, J.M., REAL, C. et SORIA, J., Solid State lonics $32-33$ (1989) 461 